\title{
Influence of Dyadic Matching of Affect on Infant Self-Regulation
}

\author{
Daniela Noe $^{a}$ Sabine Schluckwerder ${ }^{a}$ Corinna Reck ${ }^{a, b}$ \\ ${ }^{a}$ General Psychiatry, Centre for Psychosocial Medicine, Heidelberg University Hospital, Heidelberg, and \\ ${ }^{b}$ Faculty of Psychology and Educational Sciences, Ludwig Maximilian University Munich, Munich, Germany
}

\begin{abstract}
Key Words
Mother-infant interaction · Infant self-regulation · Affective behavioural matching $\cdot$ Midrange coordination $\cdot$ Still-face paradigm $\cdot$ Post-partum depression
\end{abstract}

\begin{abstract}
Background/Aims: Affective behavioural matching during face-to-face interaction fosters the transition from mutual regulation to infant self-regulation. Optimum midrange models of mother-infant interaction hold that moderate degrees of dyadic matching facilitate infant socio-emotional development. The aim of this study was to examine which degree of dyadic matching is most beneficial for infant selfregulation. Methods: To evaluate this model, 3 groups of highly, midrange and poorly matched dyads were created from a mixed sample of 68 dyads with healthy and post-partum depressed mothers and their infants (age range $=1-8$ months, mean age $=3.9$ months). Mother-infant interactions were videotaped in the face-to-face still-face paradigm (FFSF) and micro-analytically coded. Specifically, the relation between affective behavioural matching in FFSF play and infant positive and negative affect in FFSF still face and FFSF reunion was explored. Results: Contrary to our expectation, we found a monotonous trend for all groups: the more matching in FFSF play, the more positive and less negative affect the infant showed in FFSF still face and FFSF reunion, respectively. Conclusion: The present findings further illuminate the association between different degrees of dyadic matching in early mother-infant interaction and infant self-
\end{abstract}

regulation. Further research should focus on the integration and replication of findings and conceptual approaches to further evaluate and refine the concept of midrange matching and make it applicable to therapeutic work with mothers and their infants.

(c) 2015 S. Karger AG, Basel

\section{Introduction}

Starting from infancy, interactive affect and self-regulation are issues concerning the whole lifespan $[1,2]$. Infant self-regulation develops in mutually regulated dyadic parent-infant systems [3-5], and the transition from mutual regulation to infant self-regulation is fostered by affective matching during face-to-face interactions [6]. Self-regulation in infancy includes the capacity to maintain positive states as well as the management of distress and negative states [7]. The lack of an adequate development of affect regulation is seen as a precursor of the development of insecure attachment [8] and psychopathology $[9,10]$.

Besides the influence of infant temperament and situational stress level [11-15], there is profound evidence from a number of empirical studies of the pivotal importance of specific maternal interaction patterns for the infant's regulation of affect $[4,7,16-18]$. Withdrawn and intrusive interaction styles often exhibited by depressed mothers were found to be associated with adverse infant outcomes, such as a generalized disengaged and passive affectivity and self-

\section{KARGER 125}

(c) 2015 S. Karger AG, Base

0254-4962/15/0483-0173\$39.50/0

E-Mail karger@karger.com

www.karger.com/psp
Daniela Noe

General Psychiatry, Centre for Psychosocial Medicine

Heidelberg University Hospital, Vossstrasse 4

DE-69117 Heidelberg (Germany)

E-Mail Daniela.Noe@med.uni-heidelberg.de 
regulatory style as well as insecure attachment styles [1923]. Mother-infant matching can be hampered by maternal depression [22-24]. However, findings are inconsistent. Maternal depression has been associated with diminished maternal ability to coordinate face-to-face interactions and less affective behavioural matching in general, but heightened matching of negative affective states [25-28]. Beebe et al. [29] found maternal interactive coordination to be either excessive (for affect) or insufficient (for gaze and touch) in mothers with high levels of depressive symptoms (vs. low levels). On the other hand, Cohn et al. [30] found no differences in contingencies between post-partum depressed and non-depressed dyads.

Research using micro-analytic instruments to measure children's and mothers' affective behaviours in faceto-face interaction identified interactive coordinative processes, such as synchrony and affective behavioural matching, as important aspects of interactive regulation influencing the infant's socio-emotional and cognitive development [31-34]. Affective behavioural matching is a dyadic measure of interactive coordination referring to parent and infant being in the same affective state at the same time and seems to indicate the quality of dyadic interaction $[35,36]$. Regarding coordination in mother-infant interaction in general, research suggests that moderate rather than high or low levels of dyadic matching are related to more optimal outcomes, such as attachment security [for an overview, see 13, 29]. Recent micro-analytic studies revealed only moderate proportions of matching in normal play interactions from as low as $12 \%$ [37] to $30-40 \%$ of the time [38]. Tronick and Reck [23] found an interactive matching rate of $30 \%$ for positive and social affective behavioural matching in healthy (vs. depressed) mothers and their infants. In mixed samples of mothers with and without clinical diagnoses (depression, panic disorders), similar rates were found, e.g. 35 and $36 \%$ for overall affect matches, respectively, and $21.5 \%$ for positive affect matches $[36,39]$. Contradicting theoretical assumptions of attachment theory, which emphasizes the importance of sensitive mother-infant interaction with appropriate, adequate and consistent maternal reactions to infant signals, studies on maternal responsivity [40,41], contingency [42] and interactive synchrony [20] converge on the finding that midrange levels are associated with secure infant attachment, extremely high and low rates with insecure attachment classifications. On the mother's side, midrange vocal matching [43], stimulation [44] and contingency [45] have been associated with higher maternal sensitivity. Such findings underpin that midrange degrees of certain interactive phenomena such as matching, stimulation or contingency are more beneficial for infant development than excessive or reduced degrees thereof $[13,29]$.

Against the backdrop of midrange findings, the current study examines how the infant's self-regulation behaviour is affected by moderate versus heightened and lowered levels of dyadic matching in order to test and further validate the optimum midrange model of Beebe et al. [46]. Interactive and infant self-regulation of distress is seen as a key mechanism in and antecedent of attachment security, respectively [10,47-51], and in clarifying the relation between supposedly beneficial degrees of mother-infant coordination and the infant's development of self-regulation we might contribute a further piece to the completion of pathways of attachment formation and hint at ways of intervention for dyads in which interaction is impaired.

Midrange dyadic matching is defined as an indicator of interactive regulation quality and was measured in the play phase of the face-to-face still-face paradigm (FFSF) [for an overview, see 52], an experimental approximation of everyday levels of mother-infant interactive regulation. Indicators of infant self-regulation capacities (positive and negative affect) which we assume to be the result of the infant's history of interactive regulation with its mother were examined in the still-face and reunion phases of the FFSF, respectively.

We hypothesized that children in dyads with intermediate levels of dyadic matching in a naturalistic play interaction (FFSF play) are better regulated during (FFSF still face) and after a stress induction (FFSF reunion) than children in dyads with heightened or lowered dyadic matching. We expected infants in dyads with midrange coordination levels to show more positive and less negative affect in the still-face and reunion phases, respectively. Overall, we expected U-shaped functions for infant target behaviours in relation to dyadic matching degree.

\section{Method}

\section{Participants}

The participants of this study were recruited in the course of a larger longitudinal investigation of mother-infant dyads at the psychiatric University Hospital Heidelberg from September 2001 till November 2006 [for further details, see 24]. The current study included data from the first set of data collection and comprises a sample of 68 mother-infant dyads.

In summary, $85 \%(n=58)$ of the sample $(n=68)$ were diagnosed according to the Structured Clinical Interview (SCID-I [53]) and $15 \%(n=10)$ of the sample, all inpatient depressed mothers, based on a 1-hour diagnostic session and the criteria of the ICD-10 
[54]. ICD-10 diagnoses of depression are considered to be comparable to the diagnoses of the DSM-IV [55]. The healthy control sample $(\mathrm{n}=34)$ was recruited from local maternity clinics. All healthy control mothers were diagnosed using the SCID-I to exclude mothers with current or past psychiatric disorders. Further exclusion criteria were also previous treatment in a psychotherapeutic setting and an inability to effectively communicate in German. As the video session was scheduled so that the mothers in the clinical group were in an acute state of depression, the age of the infant could not be kept constant. Nevertheless, because of the reported gender differences concerning affect regulatory competences, an equal distribution of gender in both groups was taken care of, and both groups were matched according to infant age and gender [23].

The major part of the mothers in the clinical subsample was recruited from the mother-infant unit of the psychiatric University Hospital Heidelberg, where they received inpatient $(n=28)$ or outpatient $(n=3)$ treatment. Three mothers without treatment were recruited from local maternity clinics. Eligible mothers were approached by their therapist or study staff and informed about the present study. Exclusion criteria were insufficient knowledge of the German language and a diagnosis of schizophrenia or the presence of psychotic symptoms.

All depressed mothers were diagnosed according to the ICD10. Diagnoses were made by experienced psychiatrists and psychologists working in the mother-infant unit based on 1-hour psychiatric assessments. From the second year of the study period onward, depressive participants $(n=24)$ were additionally diagnosed using the SCID-I.

Participating infants were clinically normal, had a gestational age of not less than 36 weeks and no congenital abnormalities. Infant age ranged from 1 to 8 months (mean age $=3.9$ months, $\mathrm{SD}=$ 1.9 months) with 47 male and 21 female infants.

No significant demographic differences between healthy and depressed mothers were found. Consequently demographic data are presented for the whole sample of the 68 participating mothers (table 1$)$. The mean age of mothers was 32.1 years $(\mathrm{SD}=4.3$, range $=24-43)$. The average number of children including the index child was $1.6(\mathrm{SD}=0.8$, range $=1-4)$. Forty percent $(\mathrm{n}=27)$ of the participating mothers held an academic degree, $24 \%(n=16)$ were qualified for university, and intermediate secondary school was finished by $28 \%(n=19)$ of the mothers. Three $(4 \%)$ mothers completed low level secondary school, and 1 (2\%) mother had no graduation. The sociodemographic data of our sample are comparable to those reported in previous studies [56-58].

\section{Procedures and Measures}

Assessment of Mother-Infant Interaction

Mother-infant interactions were assessed using the FFSF [52]. Assessment took place in the video laboratory of the research unit at the psychiatric University Hospital of Heidelberg. Infants were seated and secured in an infant chair facing their seated mother. One camera in the research room focused on the infant, and a second camera was aimed at the mother. A microphone hung from the ceiling above the dyads. A single image comprising a simultaneous frontal view of the mother and the infant was produced by transmitting the images from the two cameras into a video recorder through a digital timer and a split-screen generator.

The FFSF comprises 3 episodes, each lasting $2 \mathrm{~min}$. In the initial face-to-face baseline interaction, mothers are instructed to engage
Table 1. Descriptive demographic sample statistics $(n=68$ mothers)

Age of mother, years

$32.1 \pm 4.3$

Age of infant, months

$3.9 \pm 1.9$

Number of children (with index child)

$1.6 \pm 0.8$

Infant gender

Male

$47(69)$

Female

$21(31)$

Education

None

General-education secondary school

$3(4)$

Junior high school

$19(28)$

University entrance diploma

$16(24)$

Academic degree

$27(40)$

Psychiatric diagnosis

$34(50)$

Results are presented as means \pm SD or numbers with percentages in parentheses.

with their infant in a 'normal' manner, playing with them without the aid of toys and without using a pacifier. This is followed by the still-face episode. Here, mothers are instructed to keep a still-face. This interruption of the mother-infant contact inhibiting interactive regulation serves as an experimental manipulation of maternal emotional unavailability and rejection. The final episode is the reunion episode, in which mothers are instructed to re-engage in face-to-face play with their infant.

In the current study we utilize the play phase for the measurement of mother-infant matching and the still-face and reunion phases for the evaluation of infant self-regulation capacity. The reunion episode poses an affectively complex and demanding regulatory task to the infant and is apt to explore how mother-infant dyads cope with emotional distress; it is commonly characterized by a carry-over of negative affect from the still-face episode [15]. It affords a twofold coping effort of the infant: first, a rebound of positive affect with the resumption of maternal interactive behaviour [59] and, secondly, the carry-over of negative affect from the still-face phase [15].

\section{Coding of Mother-Infant Interactions}

Infants' and mothers' behaviours were coded using the Noldus Observer Video-Pro ${ }^{\circledR}$ coding system - a system which inspects and analyses analogue and digital video recordings on a frame-byframe basis - and based on the micro-analytical Infant and Caregiver Engagement Phases (ICEP) [60]. The ICEP system includes a set of mutually exclusive interactive engagement phases for both the infant and mother. The phases combine information from the infant's/mother's face, direction of gaze and vocalizations. The ICEP engagement phases for the infant are: negative engagement (further divided into withdrawn and protest), object/environment engagement, social monitor and social positive engagement. ICEP codes for the mother are: negative engagement (further divided into withdrawn and hostile/intrusive), non-infant-focused engagement, social monitor/no vocalizations or neutral vocalizations, social monitor/positive vocalizations, and social positive engagement. 
Table 2. Interrater reliability in a randomly chosen subsample of $\mathrm{n}=17$ dyads

Cohen's $\kappa^{\mathrm{a}} \quad 95 \% \mathrm{CI}$

\begin{tabular}{lll} 
Infant engagement phases & & \\
$\quad$ Protest & 0.75 & $0.69-0.81$ \\
Object/environment engagement & 0.75 & $0.68-0.80$ \\
$\quad$ Social monitor & 0.83 & $0.78-0.88$ \\
$\quad$ Social positive engagement & 0.85 & $0.77-0.91$ \\
Maternal engagement phases & & \\
$\quad$ Non-infant-focused engagement & 0.82 & $0.53-1.00$ \\
$\quad$ Social monitor/neutral vocalizations & 0.72 & $0.66-0.80$ \\
$\quad$ Social monitor/positive vocalizations & 0.79 & $0.74-0.86$ \\
$\quad$ Social positive engagement & 0.73 & $0.65-0.80$ \\
\hline
\end{tabular}

$\mathrm{CI}=$ Confidence interval.

${ }^{\mathrm{a}} \mathrm{p}<0.001$.

Table 3. Descriptive statistics of groups based on proportion of matching in FFSF play

\begin{tabular}{lllll}
\hline Groups & Mean & Min. & Max. & Cases \\
\hline Low matching & 0.07 & 0.00 & 0.14 & 29 \\
Midrange matching & 0.28 & 0.15 & 0.45 & 25 \\
High matching & 0.69 & 0.51 & 0.98 & 14 \\
\hline Total & 0.27 & 0.00 & 0.98 & 68 \\
\hline
\end{tabular}

\section{Reliability of ICEP Phases}

Independent raters scored all mother-infant interactions. Coders were blind to the hypotheses of the study and to maternal and infant characteristics. Infant and maternal ICEP phases were coded twice. In order to ensure a reliable use of the ICEP codes, the raters were required to review didactic material, become familiar with the manual, and rate and review several videotapes together with the trainer. Rating discrepancies between the coder and the trainer were discussed and resolved during training. To assess interrater reliability, $25 \%$ of the videos $(\mathrm{n}=$ 17 dyads) were randomly selected and coded by 2 independent and trained coders. Mean Cohen's $\kappa$ was used as a measure of interrater reliability and was 0.79 for the infant codes and 0.77 for the mothers' codes. These $\kappa$-values are similar to those reported in previous studies $[60,61]$. The $\kappa$-values for the individual infant and maternal engagement phases are listed in table 2 . In the randomly chosen subsample of 17 dyads, 'negative engagement' and 'withdrawn' were never coded for the infant, as a result of which it was not possible to compute a $\kappa$-score for these codes. Similarly, the codes 'negative engagement', 'hostile/intrusive' and 'withdrawn' were not assigned in the subsample of mothers, so that it again was not possible to calculate $\kappa$-values. In the previous training, raters reached a reliability $>0.7$ for all scales.

\section{Diagnostic Interviews}

For diagnosing and allocating mothers to the clinical treatment or control group, 2 standard diagnostic interviews were administered: the SCID-I, a semistructured, economical and reliable instrument for diagnosing axis 1 syndromes and disorders according to the criteria of the DSM-IV [53], and the ICD-10 [54]. ICD-10 diagnoses were based on a 1-hour diagnostic interview session by an experienced psychiatrist or psychologist who worked in the mother-infant unit, and reported symptoms were classified in line with ICD-10 criteria. However, the diagnoses of these standard diagnostic instruments are considered to be comparable, especially for ICD-10 diagnoses depressive episode (F32) and receding depressive episode (F33) and the DSM-IV diagnosis major depression episode, relevant to our study [55].

\section{Sociodemographic Data}

Each participant completed an information sheet assessing sociodemographic data such as age, number of children and educational level. For each patient, the SCID-I or the ICD-10 and the sociodemographic questionnaire were administered and applied by the same experienced clinicians. Questionnaires were administered to inpatient depressed mothers during the first week of their hospitalization or outpatient treatment at the clinic. Healthy mothers were interviewed and administered the questionnaires during a visit to the laboratory.

The study protocol was approved by the independent ethics committee of the University Medical Faculty, Heidelberg. Patient confidentiality was in no way breached. Written informed consent was obtained after study procedures had been fully explained.

\section{Data Reduction and Dependent Measures}

Dyadic Measure: Proportion of Matching

Coordination was operationalized as positive affective behavioural matching. Matching states were defined as the simultaneous exhibition of the same affective-behavioural state by the mother and her infant [38]. A positive match is characterized by infant and mother being in social play with the dominant affect quality being positive. Specific infant and maternal engagement phases were defined as positive engagement categories: infant social positive engagement, infant social monitor, mother social positive engagement, mother social monitor/positive vocalization. We considered it important to conduct the analyses without the neutral maternal code 'social monitor/neutral vocalizations', since the frequent occurrence of this code may indicate less sensitive maternal interactive behaviour (e.g. criteria for social monitor/neutral vocalizations is the absence of a positive tone while speaking with the infant). The neutral infant code 'infant social monitor', which is based on the infant's direction of gaze, can be seen as a clearly positive interaction signal; infant gaze avoidance is seen as a risk factor in later cognitive development [62].

The proportion of matching was calculated by the total time matching occurred in FFSF play divided by the total length of the episode. The resulting degrees of matching proportions were then divided into 3 groups: high, midrange and low matching dyads (table 3). This 3-level classification formed the independent variable of our analyses. In order to create a window for the midrange group, we drew upon the average matching rate in normal mother-infant interactions of $30 \%$ reported in the literature [38] as an anchor value and added 15\% around this anchor. The resulting cut-off values for the midrange group are 0.15 and 0.45 . 
These are approximately congruent with the range of matching proportions reported in Tronick and Cohn [38] from 12 to 40\% of the time.

Dependent Infant Measures

Infant parameters were assessed in FFSF still face and FFSF reunion. Infant positive affect was composed of 'infant social positive engagement' and 'infant social monitor'; 'infant withdrawn' and 'infant protest' were merged into infant negative affect. The proportion of time the infant showed target affective behaviours was calculated by dividing the total time each summed up combination of codes occurred in each episode by the total length of FFSF still face and FFSF reunion, respectively.

\section{Covariates}

Given the risk and demographic status of the sample and known associations between these and target parameters, a set of covariates was introduced to control for their effects $[22,23,48$, 61]. Covariates included the categorical variables diagnosis of depression, infant gender and maternal education level, and the continuous variables age of infant, age of mother and number of children. Variables with more than 2 values were dichotomized for evaluation as potential confounding variables. Age of infant was dichotomized by median split (median $=3.6$ months). So was age of mother (median $=32.5$ years). Number of children was dichotomized by 1 versus $>1$ children, and education level of mother was dichotomized by academic versus other level of education.

\section{Statistical Analyses}

After creating the independent variable by dividing the data into 3 groups (dyads with high, midrange and low matching) based on proportion of matching in the first episode, the ICEP data were tested with 2 sets of analyses. All analyses were conducted with an $\alpha$-level of 0.05 . Analyses of dyadic behaviour involved FFSF play whereas analysis of infant behaviours involved FFSF still face and FFSF reunion.

Analysis 1: Adjustment for Confounders Based on Analyses of Covariance

Major dependent variables of this study were tested after potential effects of confounding variables (depression diagnosis, age of mother, age of infant, number of children, infant gender, maternal level of education) had been controlled for. For this purpose, analyses of covariance (ANCOVAs) were applied (SPSS procedure 'univariate' [63]) which basically test for between-group differences over regression residuals, i.e. after covariates have been partialled out [64]. However, ANCOVAs are based on a large range of assumptions: Assumptions such as homogeneity of variance were tested with the Levene test [65]; normality of residuals was checked graphically, i.e. they were plotted against normal values, 'residual normal QQ plots', with the expectation to scatter around a 45-degree line and 'residual against predicted plot' with the expectation to scatter around a horizontal line. To test the homogeneity-ofslope assumption, the interaction term of covariate and independent variable was included in the model (if $p \leq 0.05$, the term was left in the model). All tests are strictly exploratory, i.e. without adjustment for type I error. The overall idea to apply ANCOVAs was that the inclusion of covariates may reduce the total amount of error variance and thus increase statistical power to detect impairments in dyads with a depressed mother.

Dyadic Matching of Affect
Analysis 2: Calculation of Proportions of Time of Infant and Maternal Behaviour

Proportions of time of infant target behaviours in FFSF still face and FFSF reunion were calculated depending on the amount of matching in FFSF play, i.e. high (HMG), midrange (MMG) or low matching groups (LMG). Global group differences were tested with a rank analysis of variance, and differences in change values from FFSF play to FFSF still face and FFSF reunion were subjected to pairwise comparisons based on exploratory Mann-Whitney U tests. We opted for these nonparametric procedures due to the skewness of the matching proportions' distribution.

\section{Results}

\section{Analysis of Matching}

Data Distribution of Matching Proportions

Matching of positive affect averages $27 \%$ of the time in the 68 dyads of our sample (table 3 ). The median of the right-skewed distribution is at 0.18 , the standard deviation is 0.25 . Matching proportions ranged from 0 to $98 \%$.

\section{Groups of High, Midrange and Low Matching}

The LMG is comprised of 29 cases with an average matching rate of $7 \%$ of the time (range $0.00-0.14$; table 3 ). The 25 dyads in the MMG are matched for $28 \%$ of the time (cut-off values $0.15-0.45)$. In the HMG $(n=14)$, there occurs more than twice as much matching as in the MMG (69\%, range 0.51-0.98).

\section{Effects of Confounders}

We found no significant effects of possible confounding variables (diagnosis of depression, age of infant, age of mother, number of children, gender of infant and education level of mother) on infant target parameters. In other words, after partialling out possible confounders, the matching groupings remain. Consequently covariates are not regarded as confounding parameters for the grouping into high, low and midrange matchers.

\section{Hypothesis 1: Proportion of Infant Positive Affect}

Contrary to our expectation of a more positive infant outcome in midrange dyads, we found a monotonous trend: the more matching in FFSF play, the more positive affect the infant showed in FFSF still face and FFSF reunion, respectively (table 4). Infants in the HMG spent about twice as much time (still face 51\%; reunion $62 \%$ ) in positive engagement as infants in the LMG (still face 23\%; reunion 29\%) or MMG (still face 
Table 4. Proportions of time of infant and maternal behaviour in FFSF episodes depending on the amount of matching in FFSF play

\begin{tabular}{|c|c|c|c|c|c|c|c|c|c|}
\hline & \multicolumn{3}{|c|}{ Low matching $(\mathrm{n}=29)$} & \multicolumn{3}{|c|}{ Midrange matching $(\mathrm{n}=25)$} & \multicolumn{3}{|c|}{ High matching $(\mathrm{n}=14)$} \\
\hline & play & still-face & reunion & play & still-face & reunion & play & still-face & reunion \\
\hline Infant negative & $0.10 \pm 0.05^{\mathrm{b}}$ & $0.21 \pm 0.06$ & $0.20 \pm 0.05^{\mathrm{b}}$ & $0.07 \pm 0.03^{c}$ & $0.14 \pm 0.05$ & $0.16 \pm 0.05$ & $0.00 \pm 0.00$ & $0.04 \pm 0.02$ & $0.06 \pm 0.04$ \\
\hline Infant protest & $0.09 \pm 0.05^{\mathrm{b}}$ & $0.20 \pm 0.07$ & $0.19 \pm 0.06^{\mathrm{b}}$ & $0.06 \pm 0.03^{c}$ & $0.14 \pm 0.05$ & $0.14 \pm 0.05$ & $0.00 \pm 0.00$ & $0.04 \pm 0.02$ & $0.06 \pm 0.04$ \\
\hline Infant withdrawn & $0.01 \pm 0.01$ & $0.01 \pm 0.01$ & $0.02 \pm 0.01$ & $0.01 \pm 0.01$ & & $0.01 \pm 0.01$ & $0.00 \pm 0.00$ & & $0.00 \pm 0.00$ \\
\hline Infant positive & $0.16 \pm 0.03^{\mathrm{a}, \mathrm{b}}$ & $0.23 \pm 0.05$ & $0.29 \pm 0.04^{\mathrm{b}}$ & $0.41 \pm 0.04^{\mathrm{c}}$ & $0.41 \pm 0.06$ & $0.33 \pm 0.05^{c}$ & $0.83 \pm 0.03$ & $0.51 \pm 0.06$ & $0.62 \pm 0.08$ \\
\hline Infant social monitor & $0.14 \pm 0.03^{\mathrm{a}, \mathrm{b}}$ & $0.22 \pm 0.05$ & $0.25 \pm 0.04^{\mathrm{b}}$ & $0.36 \pm 0.04^{c}$ & $0.04 \pm 0.06$ & $0.29 \pm 0.04^{\mathrm{c}}$ & $0.67 \pm 0.06$ & $0.47 \pm 0.05$ & $0.49 \pm 0.07$ \\
\hline Infant social positive & $0.03 \pm 0.01^{\mathrm{b}}$ & $0.01 \pm 0$ & $0.04 \pm 0.01^{\mathrm{b}}$ & $0.05 \pm 0.02^{\mathrm{c}}$ & $0.01 \pm 0$ & $0.04 \pm 0.01^{\mathrm{c}}$ & $0.18 \pm 0.05$ & $0.04 \pm 0.02$ & $0.14 \pm 0.04$ \\
\hline Mother negative & $0.05 \pm 0.03$ & & $0.04 \pm 0.02$ & $0.01 \pm 0.00$ & & $0.00 \pm 0.00$ & $0.00 \pm 0.00$ & & $0.00 \pm 0.00$ \\
\hline Mother hostile/intrusive & & & $0.01 \pm 0.01$ & $0.00 \pm 0.00$ & & $0.00 \pm 0.00$ & $0.00 \pm 0.00$ & & $0.00 \pm 0.00$ \\
\hline Mother withdrawn & $0.05 \pm 0.03$ & & $0.03 \pm 0.02$ & $0.01 \pm 0.00$ & & $0.00 \pm 0.00$ & $0.00 \pm 0.00$ & & $0.00 \pm 0.00$ \\
\hline Mother positive & $0.47 \pm 0.05^{\mathrm{a}, \mathrm{b}}$ & & $0.55 \pm 0.06$ & $0.67 \pm 0.05^{c}$ & & $0.69 \pm 0.05$ & $0.82 \pm 0.03$ & & $0.72 \pm 0.05$ \\
\hline $\begin{array}{c}\text { Mother social monitor/ } \\
\text { positive vocalizing }\end{array}$ & $0.42 \pm 0.05^{\mathrm{a}, \mathrm{b}}$ & & $0.49 \pm 0.05$ & $0.56 \pm 0.04^{\mathrm{c}}$ & & $0.59 \pm 0.05$ & $0.71 \pm 0.03$ & & $0.62 \pm 0.04$ \\
\hline Mother social positive & $0.05 \pm 0.01^{\mathrm{a}, \mathrm{b}}$ & & $0.06 \pm 0.02^{b}$ & $0.12 \pm 0.03$ & & $0.11 \pm 0.04$ & $0.12 \pm 0.03$ & & $0.11 \pm 0.03$ \\
\hline
\end{tabular}

Data are expressed as means \pm SE. Test for global group differences: rank analysis of variance [65]. All comparisons are strictly exploratory, $\mathrm{p}$ values $\leq 0.05$ denote an exploratory difference. Last columns for pairwise comparisons based on exploratory Mann-Whitney tests and if $p$ values $\leq 0.05$ then: ${ }^{\mathrm{a}} \mathrm{p} \leq$ 0.05 , low vs. midrange; ${ }^{b} \mathrm{p} \leq 0.05$, low vs. high; ${ }^{c} \mathrm{p} \leq 0.05$, midrange vs. high. During the still-face episode, mothers were instructed to interrupt the interaction with their infant. Therefore it is not possible to present maternal behaviour for this phase.

Table 5. Significant group differences for LMG, MMG and HMG according to FFSF episode

\begin{tabular}{lcc}
\hline & Mann-Whitney U & $\mathrm{p}$ \\
\hline $\begin{array}{l}\text { Infant positive affect } \\
\text { Still-face }\end{array}$ & \\
$\quad$ LMG vs. MMG & 224.5 & $<0.01$ \\
$\quad$ LMG vs. HMG & 79.5 & 0.0002 \\
Reunion & & \\
$\quad$ LMG vs. HMG & 74.5 & 0.001 \\
$\quad$ MMG vs. HMG & 76.0 & 0.004 \\
Play to reunion changes & 171.5 & 0.001 \\
$\quad$ LMG & 47.5 & $<0.001$ \\
$\quad$ MMG & & \\
\hline Infant negative affect & & \\
Reunion & 100.5 & 0.009 \\
\hline LMG vs. HMG & & \\
\hline
\end{tabular}

41\%; reunion 33\%). During FFSF still face, the LMG differs from the MMG $(\mathrm{U}=224.5 ; \mathrm{p}<0.01)$ and $\mathrm{HMG}$ $(\mathrm{U}=79.5 ; \mathrm{p}=0.0002$ ) significantly (for an overview, see table 5). In FFSF reunion, the HMG differs from the LMG $(\mathrm{U}=74.5 ; \mathrm{p}=0.001)$ and MMG $(\mathrm{U}=76$; $\mathrm{p}=$ $0.004)$ significantly. For the major part of the time spent in positive affect, infants directed their neutral attention to mothers (LMG 25\%, MMG 29\% and HMG $49 \%$ ), and for a lesser proportion of time they showed joyful positive affect (LMG 4\%, MMG 4\% and $\mathrm{HMG}$ $14 \%)$.

We found a significant difference between LMG and the other groups for the change from play to reunion: proportion of time spent in positive engagement almost doubles from play to reunion for the LMG (from 16 to $29 \% ; \mathrm{U}=171.5 ; \mathrm{p}=0.001$ ), whereas it diminishes in the MMG (from 41 to $33 \%$; $\mathrm{U}=47.5 ; \mathrm{p}<0.001$ ) and $\mathrm{HMG}$ (from 83 to $62 \%$; $\mathrm{U}=124.5 ; \mathrm{p}=0.139$ ).

The mothers' proportion of positive affect (LMG: $47 \%$; MMG: 67\%; HMG: 82\%) differs significantly between groups for the FFSF play (LMG vs. MMG: $\mathrm{U}=219.5, \mathrm{p}=$ 0.013; LMG vs. HMG: $\mathrm{U}=55.5, \mathrm{p}<0.001 ; \mathrm{MMG}$ vs. HMG: $U=105, p=0.04$ ) whereas for the reunion their difference does not reach the level of significance. All mothers, in the reunion, spent approximately half to three quarters of the time in positive affect.

Mothers in the LMG and MMG, in the reunion episode, spent about twice as much time in positive affective states as their infants (LMG infant: $29 \%$, mother: $55 \%$; MMG infant: $33 \%$, mother: 69\%), whereas HMG mothers spent only $10 \%$ more time in positive engagement states (72\%) than their infants (62\%). Disentangling the combined affect categories, an overall picture emerges of infants directing their attention towards and looking at the mother, signalling social interest and mothers observing the infants and their activities, occasionally smiling and vocalizing positively. 
Hypothesis 2: Proportion of Infant Negative Affect

For infant negative affect, we also found a monotonous trend: MMG infants did not show the least negative affect but HMG infants did (table 4). The more mother and infant were affectively matched in the FFSF play, the less infant negative affect occurred in the FFSF still face and FFSF reunion. The overall occurrence of negative engagement is strikingly low in infant (FFSF play: $0-10 \%$; still face: $4-21 \%$; reunion: $6-20 \%$ ) as well as in maternal behaviour (FFSF play: 0-5\%; reunion: $0-4 \%$ ); in the HMG it is virtually non-occurrent. In the play phase, LMG infants spent twice as much time in negative states as mothers ( $10 \mathrm{vs.} 5 \%)$, whereas in reunion the proportion of time infants spent in negative affectivity is 5 times as high as their mothers' (20 vs. $4 \%$ ). In the MMG, the relation is 1 vs. $7 \%$ (mother vs. infant) for the play phase; in reunion, MMG mothers show no negative affect whereas the infants' proportion is $16 \%$. In the HMG, negative affect in the form of protest is exclusively shown by infants in the reunion phase $(6 \%)$.

There were no significant group differences in FFSF still face, but HMG and LMG differed significantly for negative engagement in FFSF reunion $(\mathrm{U}=100.5$; $\mathrm{p}=$ $0.009)$. Infant negative engagement in the HMG merely yielded floor effects (6\%). In contrast, infants in the MMG (16\%) and LMG (20\%) spent about 3 times as much time in negative engagement states in FFSF reunion.

\section{Discussion}

This study addresses the question which degree of dyadic matching in mother-infant interaction is most beneficial for the infant's development of self-regulation. Contrary to our expectations of a more positive infant outcome in midrange dyads, however, the results indicate that higher degrees of affective matching are related to better self-regulation: infants in highly matched dyads showed more positive affect and less negative affect than infants in dyads with low or midrange matching.

Infants in highly matched dyads spent about twice as much time in positive affective states as infants in low and midrange matching dyads, while negative affect was only marginally present in the high matching dyads, but reached substantial proportions of time in the low and midrange matching dyads. Altogether this resembles the mixed pattern of positive and negative affect expected for the FFSF reunion [15]. Converging with Weinberg and Tronick [15] we also found more negative affective be-

Dyadic Matching of Affect haviours in the still face and reunion phases than in play in all of the dyads, indicating that the distressful experience of maternal emotional separation is not easily repaired by maternal resumption of interaction. Concerning positive affect, we also found the previously reported rebound in reunion and (for midrange and high matching infants) also typically lower proportions than in play [39]. Infants in low matching dyads double their proportions of negative as well as positive affective states from play to reunion, a picture of an overall heightening of affective behaviour. In midrange and highly matched infants, negative affect rises from play to reunion, while positive affect lowers.

Our result of a total average matching rate of $27 \%$ in the play phase of the FFSF is in accordance with results of a matching rate of $30 \%$ in a regular play situation [38] and underpins findings and hypotheses of moderate rates of matching in everyday mother-infant interaction in mixed and non-clinical samples $[36,38]$. This value is also approximated by our MMG average rate of matching of $28 \%$. Low matching dyads, in contrast, spent a mere $7 \%$ of the time in shared positive affect, which is comparable to a positive matching rate of $10 \%$ during normal play for cocaine- and opiate-exposed dyads [60]. High matchers in our sample shared positive states for a very high proportion of $69 \%$ of the time, which to our knowledge has not been reported so far.

The mothers' exhibition of positive affect shows a similar trend in reunion as that of infants with mothers from poorly matched dyads showing the lowest proportions and mothers from highly matched dyads showing the highest proportions. However, these differences were not significant. Generally, mothers showed more positive and less negative affect in the reunion phase than their infants, which is in accordance with Fogel's concept of framing, i.e. the adult's capacity to create an atmosphere in which the interaction can continue, and hints at their efforts to draw the infant into positive states [66].

High matching mothers in our sample invest more positive affect and also seem to be more successful, supporting concepts of mutual emotional contagion in mother-infant interaction [16]. Shared positive affect and positive mutuality foster infant cognitive and socio-emotional development whereas chronic sharing of negative affect is conducive to vicious circles and escalation of negativity $[1,13,26,36,38,61]$. Furthermore, Emde [67] has described the optimal mother as one who not only reacts sensitively to the infant's expression of distress but is also emotionally available for and capitalizes upon the infant's expression of positive affect. 
The overall occurrence of negative affect in mothers as in infants was strikingly low. On the one hand this very low negativity in maternal behaviours may result from a deficient graduation of negative-behaviour codes in the ICEP system. Here, the various engagement codes are defined very strictly; it is, for instance, not possible to code mere maternal overstimulation. In line with this, the ICEP data of a large sample of cocaine-exposed and nonexposed infants indicate a low representation of negative ICEP codes [60]. Tronick and his working group reported a $0.6 \%$ occurrence of negative engagement on the part of the mother in the play episode and a $1.5 \%$ occurrence in the reunion episode. Infants protested in $3.5 \%$ of the play and $15 \%$ of the reunion episodes. Our finding of very low proportions of negative mothers' behaviour converges with those of Weinberg et al. [61], who also found maternal negative engagement in social interaction with the infant to be extremely rare in normal samples, whereas in studies with risk samples of cocaine-exposed or depressed mothers, negative engagement was evident under stressful conditions like FFSF reunion $[39,60]$. In the current study, maternal positive affect increased in low and midrange matching dyads from play to reunion and diminished in the mothers with high matching. In order to disentangle this mixed picture, future studies should include qualitative surveys on mothers' subjective experiences of the experimental perturbation of the still-face and parenting representations.

Various theories capitalize upon the process of flexibly transitioning between positive and negative states as a crucial aspect of successful affect regulation in parentinfant dyads $[1-3,38]$. As distress is an unavoidable phenomenon of human interaction, the modulation, toleration and endurance of experiences of negative affect can be seen as a developmental task with the successful regulation of experiences thereof fostering a sense of self-efficacy [7]. The non-occurrence of negative affect disenables the infant to experience an interactive repair or autonomous mastery of negative arousal and the ensuing transition from a negative to positive affective state. In the light of these arguments, it becomes even more evident that long-term studies are needed. They would allow for determining whether a highly coordinative interactional style combined with a very low occurrence of negative affect is beneficial in the long run, i.e. develops into secure attachment styles, or whether, as Beebe's research and interpretation of tight, 'rather obligatory than optional' coupling, as rigidity indicates, insecure attachment styles are the more probable developmental outcome [13].
As a strength of the current study, its effort at systematic integration of individual levels with dyadic analyses has to be pointed out, as advocated by Diamond and Aspinwall [2] in their conclusive review on emotion regulation from a lifespan perspective. In basing our analyses on a dyadic measure of matching, infant and mother contributions to the interactive process are equally acknowledged, and the demands of a conceptualization of development from a systems point of view are met, emphasizing the co-construction of interaction $[3,66]$. Another strength of the present study is the use of micro-analytically coded, observational data for investigating an issue relevant to the formation of attachment security [68]. Other studies in the midrange tradition mainly drew upon global measures of maternal sensitivity and attachment at a later point of time (12 months or older) as their criteria [1, 13, 40]. Furthermore, we evaluated relevant covariates, and our finding that a diagnosis of depression has no influence on infant self-regulation after a stressful episode of maternal separation converges with null findings for the dependence of maternal sensitivity and interactive behaviour of clinical diagnosis [39, 69]. It contradicts findings that dyads with depressed mothers are impaired in matching their affective states and that infants of depressed versus those of non-depressed mothers showed less distress behaviours during the still-face condition and also showed fewer positive as well as fewer negative behaviours during reunion [70-72]. Further research is needed to disentangle the adverse effects of post-partum depression on infant development.

Finally, in the light of our findings the midrange model of dyadic matching cannot be fully rejected. What was shown was that regarding the matching of positive affect, there is a monotonous trend towards higher matching correlated with better infant affect regulation. Further analyses, however, with another operationalization of midrange matching might confirm midrange hypotheses, e.g. coordination conceptualized as tightness of coupling with reaction latencies as operationalization. Various windows for reaction latencies in motherinfant interaction have been found and discussed in the literature. Values range from very short latencies of $\leq 1 \mathrm{~s}$ [73], over 2-3 s $[29,41,74]$ up to $3-5 \mathrm{~s}$ [75] and 5-7 s [76] as limits for the experience of contingency for infants. Applying the concept of midrange matching to this range of reaction latencies, midrange maternal reaction latency could then serve either as a grouping criterion for a new independent variable or, in relation to the existing groups, the reaction latencies of the high match- 
ers could be tested. High matching dyads might as well turn out to be midrange dyads with reaction latencies as the criterion.

\section{Limitations of the Study}

Several limitations of the present study should be noted. First, although typical of many micro-analytical studies, our sample size was relatively small. Additionally, the analysis of negative affect was limited. This may be due to the reasons stated above, or there has been a tendency for the majority of relevant studies to code a low degree of negativity. The frequency with which negativity is coded would appear to vary according to the coding system used. Studies which have coded a greater degree of negative interaction behaviour have either employed macroanalytical approaches $[19,31,32]$ in which low negativity thresholds (e.g. maternal intrusiveness) were applied or they have coded specific individual behaviours, such as smiles or gaze aversions, in an event-based manner. In contrast to participating mothers, we did not apply a diagnostic instrument for the infants. This should be included in further studies in order to evaluate infant characteristics in more detail. Another limitation is the variation of infant age which is due to the scheduling criterion for the video session being the mother's state of acute depression. ANCOVAs showed that the matching groups' effects remained after controlling for infant age; the current study's findings can be seen as a hint for the independence of the effects of mother-infant coordination of infant age. Due to the exploratory nature of our analysis of covariates, further support for this finding is needed.

\section{Conclusion}

The present findings further illuminate the effect of different degrees of dyadic matching in early mother-infant interaction on infant self-regulation in a mixed sample of depressed and non-depressed mothers and their infants. Infant self-regulation reflects the developing attachment style and can thus serve as a valuable diagnostic concept for early preventive interventions [47]. Our finding that a maternal diagnosis of depression has no influence on infant self-regulation is indicative of focussing on behavioural coordinative therapeutic interventions for mothers together with their infants in order to capitalize on this dyadic resource and to prevent developmental disorders in infants $[23,77]$.

Longitudinal studies are needed to determine the longterm consequences of midrange matching on behavioural and physiological infant self-regulation and, ultimately, attachment style formation. Future studies should investigate other aspects of affect regulation not examined in this study such as infant temperament or psychiatric disorders [14]. Apart from behavioural measures, they should include physiological or neurological reactions [36] and account for possible differences in underlying arousal between groups whose behavioural reactions seem similar [15]. Future studies should also include different situational contexts for the assessment of maternal and infant behaviours in order to extend the external validity of findings. In relation to low maternal negativity, research should be extended to more naturalistic, e.g. home, settings in which mothers may feel less observed.

The wide range of idiosyncratic research concepts and approaches complicates communication and comparison of empirical findings. For the development and evaluation of concrete and specific interventions for mothers and their infants generalizable and transferable findings are needed. Further research should focus on the integration and replication of findings and conceptual approaches to further evaluate and refine the concept of midrange matching and make it applicable to therapeutic work with mothers and their infants.

\section{Acknowledgements}

This study was supported by a grant from the Program of Research Support at the University Medical Faculty, Heidelberg (funding period: 2003-2004).

\section{Disclosure Statement}

There are no competing interests, including specific financial interests, relationships, or affiliations relevant to the subject of the study.
References
1 Beebe B, Jaffe J, Lachmann F, Feldstein S, Crown C, Jasnow M: Systems models in de- velopment and psychoanalysis: the case of vo- cal rhythm coordination and attachment. In- fant Ment Health J 2000;21:99-122.
$\checkmark 2$ Diamond LM, Aspinwall LG: Emotion regu- lation across the life span: an integrative per- spective emphasizing self-regulation, positive affect, and dyadic processes. Motiv Emot 2003;27:125-156.
$\checkmark 3$ Tronick EZ: Emotions and emotional com- munication in infants. Am Psychol 1989;44: 112-119. 
4 Kopp CB: Antecedents of self-regulation: a developmental perspective. Dev Psychol 1982;18:199-214.

5 Fonagy P, Gergely G, Jurist EL, Target M: The social biofeedback theory of affect-mirroring: the development of emotional self-awareness and self-control in infancy; in Fonagy $\mathrm{P}$, Gergely G, Jurist EL, Target M (eds): Affect Regulation Mentalization, and the Development of the Self. New York, Other Press, 2002, pp 145-202.

6 Gianino A, Tronick EZ: The mutual regulation model: the infant's self and interactive regulation and coping and defensive capacities; in Field T, MacCabe P, Schneiderman N (eds): Stress and Coping. Hillsdale, Erlbaum, 1988, pp 47-68.

7 Kopp CB: Regulation of distress and negative emotions: a developmental view. Dev Psychol 1989;25:343-354.

8 De Oliveira CA, Neufeld Bailey H, Moran G, Pederson DR: Emotion socialization as a framework for understanding the development of disorganized attachment. Soc Dev 2004; 13:437-467.

-9 Calkins SD, Fox NA: Self-regulatory processes in early personality development: a multilevel approach to the study of childhood social withdrawal and aggression. Dev Psychopathol 2002;14:477-498

10 Cummings EM, Davies P: Emotional security as a regulatory process in normal development and the development of psychopathology. Dev Psychopathol 1996;8:123-139.

11 Braungart-Rieker JM, Garwood MM, Powers BP, Notaro PC: Infant affect and affect regulation during the still face paradigm with mothers and fathers: the role of infant characteristics and parental sensitivity. Dev Psychol 1998;34:1428-1437.

$\checkmark 12$ Fox N, Calkins SD: The development of selfcontrol of emotion: intrinsic and extrinsic influences. Motiv Emot 2003;27:7-26.

-13 Jaffe J, Beebe B, Feldstein S, Crown C, Jasnow M: Rhythms of dialogue in infancy: coordinated timing in development. Monogr Soc Res Child Dev 2001;66:1-132.

14 Rothbart MK, Derryberry D: Development of individual differences in temperament; in Lamb ME, Brown AL (eds): Advances in Developmental Psychology. Hillsdale, Erlbaum, 1981, vol 1, pp 37-86.

15 Weinberg MK, Tronick EZ: Infant affective reactions to the resumption of maternal interaction after the Still-Face. Child Dev 1996;67: 905-914.

16 Beebe B, Lachmann F, Jaffe J: Mother-infant interaction structures and presymbolic selfand object representations. Psychoanal Dialogues 1997;7:133-182.

17 Lyons-Ruth K, Block D: The disturbed caregiving system: relations among childhood trauma, maternal caregiving, and infant affect and attachment. Infant Ment Health J 1996 17:257-275

18 Weinberg MK, Tronick EZ: The impact of maternal psychiatric illness on infant devel- opment. J Clin Psychiatry 1998;59(suppl 2): 53-61.

19 Field T, Hernandez-Reif M, Diego M: Intrusive and withdrawn depressed mothers and their infants. Dev Rev 2006;26:15-30.

20 Isabella RA, Belsky J, von Eye A: Origins of infant-mother attachment: an examination of interactional synchrony during the infant's first year. Dev Psychol 1989;25:12-21.

21 Isabella RA, Belsky J: Interactional synchrony and the origins of infant-mother attachment: a replication study. Child Dev 1991;62:373384.

22 Reck C, Hunt A, Fuchs T, Weiss R, Noon A, Moehler E: Interactive regulation of affect in postpartum depressed mothers and their infants: an overview. Psychopathology 2004;37: 272-280.

23 Tronick EZ, Reck C: Infants of depressed mothers. Harv Rev Psychiatry 2009; 17:147156.

24 Reck C, Noe D, Stefenelli U, Fuchs T, Cenciotti F, Stehle E: Interactive coordination of currently depressed inpatient mothers and their infants during the postpartum period. Infant Ment Health J 2011;32:542-562.

25 Cohn JF, Tronick EZ: Specificity of infant's response to mother's affective behaviour. J Am Acad Child Adolesc Psychiatry 1989;28: 242-248.

26 Field T, Healy B, Goldstein S, Guthertz M: Behaviour-state matching and synchrony in mother-infant interactions of nondepressed versus depressed dyads. Dev Psychol 1990;26: 7-14.

27 Field T: Infants of depressed mothers. Dev Psychopathol 1992;4:49-66.

28 Zlochower AJ, Cohn JF: Vocal timing in faceto-face interaction of clinically depressed and nondepressed mothers and their 4-month-old infants. Infant Behav Dev 1996;19:371-374.

29 Beebe B, Jaffe J, Buck K, Chen H, Cohen P, Feldstein S: Six-week postpartum maternal depressive symptoms and 4-month motherinfant self- and interactive contingency. Infant Ment Health J 2008;29:442-471.

30 Cohn J, Campbell S, Matias R, Hopkins J: Face-to-face-interactions of postpartum depressed and nondepressed mother-infant pairs at 2 months. Dev Psychol 1990;26:15-23.

31 Feldman R: Parent-infant synchrony and the construction of shared timing: physiological precursors, developmental outcomes, and risk conditions. J Child Psychol Psychiatry 2007;48:329-354.

32 Feldman R: Parent-infant synchrony: biological foundations and developmental outcomes. Curr Dir Psychol Sci 2007;16:340-345.

- 33 Feldman R, Greenbaum CW, Yirmiya N: Mother-infant affect synchrony as an antecedent of the emergence of self-control. Dev Psychol 1999;35:223-231.

34 Harrist AW, Waugh RM: Dyadic synchrony: its structure and function in children's development. Dev Rev 2002;22:555-592.

35 Lowe JR, MacLean PC, Duncan AF, Aragon C, Schrader AM, Caprihan A, Phillips JP: As- sociation of maternal interaction with emotional regulation in 4- and 9-month infants during the Still Face Paradigm. Infant Beh Dev 2012;35:295-302.

-36 Moore GA, Calkins SD: Infants' vagal regulation in the Still-Face Paradigm is related to dyadic coordination of mother-infant interaction. Dev Psychol 2004;40:1068-1080.

37 Lester BM, Hoffman J, Brazelton T: The rhythmic structure of mother-infant interaction in term and preterm infants. Child Dev 1985;56:15-27.

38 Tronick EZ, Cohn JF: Infant-mother face-toface interaction: age and gender differences in coordination and the occurrence of miscoordination. Child Dev 1989;60:85-92.

39 Weinberg MK, Beeghly M, Olson KL, Tronick EZ: Effects of maternal depression and panic disorder on mother-infant interactive behaviour in the face-to-face still-face paradigm. Infant Ment Health J 2008;29:472-491.

40 Lewis M, Feiring C: Infant, mother, and mother-infant interaction behaviour and subsequent attachment. Child Dev 1989;60: 831-837.

41 Leyendecker B, Lamb ME, Fracasso MP, Schölmerich A: Playful interaction and the antecedents of attachment: a longitudinal study of Central American and EuroAmerican mothers and infants. Merrill Palmer Q 1997;43:24-47.

42 Gergely G, Watson JS: Early socio-emotional development: contingency perception and the social-biofeedback model; in Rochat $\mathrm{P}$ (ed): Early Social Cognition: Understanding Others in the First Months of Life. Mahwah, Erlbaum, 1999, pp 101-136.

43 Hane AA, Feldstein S, Dernetz VH: The relation between coordinated interpersonal timing and maternal sensitivity in four-monthold infants. J Psycholinguist Res 2003;32:525539.

44 Roe KV, Drivas A: Reciprocity in mother-infant vocal interactions: relationship to the quantity of mothers' vocal stimulation. Am J Orthopsychiatry 1997;67:645-649.

45 Bornstein MH, Manian N: Maternal responsiveness and sensitivity reconsidered: some is more. Dev Psychopathol 2013;25:957-971.

46 Beebe B, Rustin J, Sorter D, Knoblauch S: An expanded view of intersubjectivity in infancy and its application to psychoanalysis. Psychoanal Dialogues 2003;13:805-841.

47 Braungart-Rieker JM, Garwood MM, Powers BP, Wang X: Parental sensitivity, infant affect and affect regulation: predictors of later attachment. Child Dev 2001;72:252-270.

48 Kogan N, Carter AS: Mother-infant reengagement following the still-face: the role of maternal emotional availability in infant affect regulation. Infant Behav Dev 1996;19:359-370.

49 Koulomzin M, Beebe B, Anderson S, Jaffe J, Feldstein S, Crown C: Infant gaze, head, face and self-touch at 4 months differentiate secure vs avoidant attachment at 1 year: a microanalytic approach. Attach Hum Dev 2002; $4: 3-24$. 
50 Spangler G, Grossmann KE: Biobehavioral organization in securely and insecurely attached infants. Child Dev 1993;64:1439-1450.

-51 Costa Martins E, Soares I, Martins C, Tereno S, Osorio A: Can we identify emotion overregulation in infancy? Associations with avoidant attachment, dyadic emotional interaction and temperament. Infant Child Dev 2012;21:579-595.

52 Mesman J, van Ijzendoorn MH, BakermansKranenburg MJ: The many faces of the StillFace Paradigm: a review and meta-analysis. Dev Rev 2009;29:120-162.

53 Wittchen HU, Wunderlich U, Gruschwitz S, Zaudig M: Strukturiertes klinisches Interview für DSM-IV, Achse 1 (SKID) (Structured Clinical Interview for DSM Disorders Axis I, SCID-I). Göttingen, Hogrefe, 1997.

54 World Health Organization: The ICD-10 Classification of Mental and Behavioural Disorders, ed 10. Geneva, World Health Organization, 1992.

55 Mombour W, Spitzner S, Reger KH, von Cranach M, Dilling H, Helmchen $\mathrm{H}$ : Summary of the qualitative criticisms made during the ICD-10 field trial and remarks on the German translation of ICD-10. Pharmacopsychiatry 1990;23(suppl IV):197-201.

-56 Weinberg KM, Olson KL, Beeghly M, Tronick EZ: Making up is hard to do, especially for mothers with high levels of depressive symptoms and their infant sons. J Child Psychol Psychiatry 2006;47:670-683.

-57 Feldman R, Greenbaum CW, Yirmiya N, Mayes LC: Relations between cyclicity and regulation in mother-infant interaction at 3 and 9 months and cognition at 2 years. J Appl Dev Psychol 1996;17:347-365.

-58 Feldman R: Infant-mother and infant-father synchrony: the coregulation of positive arousal. Infant Ment Health J 2003;24:1-23.

59 Gusella JL, Muir D, Tronick EZ: The effect of manipulating maternal behavior during an interaction on three- and six-month-olds' affect and attention. Child Dev 1988;59:1111-1124.
60 Tronick EZ, Messinger DS, Weinberg MK, Lester BM, LaGasse L, Seifer R: Cocaine exposure is associated with subtle compromises of infants' and mothers' social-emotional behavior and dyadic features of their interaction in the face-to-face still-face paradigm. Dev Psychol 2005;41:711-722.

61 Weinberg MK, Tronick EZ, Cohn JF, Olson KL: Gender differences in emotional expressivity and self-regulation during early infancy. Dev Psychol 1999;35:175-188.

62 Laucht M, Esser G, Schmidt M: Heterogene Entwicklung von Kindern postpartal depressiver Mütter (Heterogeneous development of children of postnatally depressed mothers). Z Klin Psychol Psychother 2002;31: 127-134

63 Norusis MJ: SPSS 16.0 Advanced Statistical Procedures Companion. Upper Saddle River, Prentice Hall, 2008.

64 Winer BJ, Brown DR, Michels KM: Statistical Principles in Experimental Design. New York, McGraw-Hill, 1991

65 Lehmann EL: Nonparametrics - Statistical Methods Based on Ranks. Upper Saddle River, Prentice Hall, 1998.

66 Fogel A: Two principles of communication: co-regulation and framing; in Nadel J, Camaioni L (eds): New Perspectives in Early Communicative Development. London, Routledge, 1993, pp 9-22.

67 Emde RN: Emotional availability: a reciprocal reward system for infants and parents with implications for the prevention of psychosocial disorders; in Goldson E (ed): Parent-Infant Relationships. Orlando, Grune \& Stratton, 1980, pp 87-115.

68 Peck SD: Measuring sensitivity moment-bymoment: a microanalytic look at the transmission of attachment. Attach Hum Dev 2003;5:38-63.
69 Carter AS, Garrity-Rokous FE, Chazan-Cohen R, Little C, Briggs-Gowan MJ: Maternal depression and comorbidity: predicting early parenting, attachment security, and toddler social-emotional problems and competencies. J Am Acad Child Adolesc Psychiatry 2001;40:18-26.

70 Field T, Hernandez-Reif M, Diego M, Feijo L Vera Y, Gil K, Sanders C: Still-face and separation effects on depressed mother-infant interactions. Infant Ment Health J 2007;28:314323.

71 Jameson P, Gelfand D, Kulcsar E, Teti D: Mother-toddler interaction patterns associated with maternal depression. Dev Psychopathol 1997;9:537-550.

72 Feldman R: Maternal versus child risk and the development of parent-child and family relationships in five high-risk populations. Dev Psychopathol 2007;19:293-312.

73 Keller H, Lohaus A, Völker S, Cappenberg M, Chasiotis A: Temporal contingency as an independent component of parenting behavior. Child Dev 1999;70:474-485.

74 Stanley C, Murray L, Stein A: The effect of postnatal depression on mother-infant interaction, infant response to the still-face perturbation, and performance on an instrumental learning task. Dev Psychopathol 2004;16:118.

75 Watson J: Detection of self: the perfect algorithm; in Parker S, Mitchell R, Boccia M (eds): Self-Awareness in Animals and Humans: Developmental Perspectives. New York, Cambridge University Press, 1994.

76 Bornstein MH, Tamis-LeMonda CS, Tal J, Ludemann P, Toda S, Rahn CW: Maternal responsiveness to infants in three societies: the United States, France, and Japan. Child Dev 1992;63:808-821.

77 Forman DR, O'Hara MW, Stuart S, Gorman L, Larsen KE, Coy KC: Effective treatment for postpartum depression is not sufficient to improve the developing mother-child relationship. Dev Psychopathol 2007;19:585-602. 